\title{
Noise shielding surrogate models using dynamic artificial neural networks
}

\author{
Francesco Centracchio* \\ Lorenzo Burghignoli ${ }^{\dagger}$ \\ Giorgio Palma \\ Ilaria Cioffi ${ }^{\S}$ umberto Iemma ${ }^{\text {II }}$ \\ Roma Tre University, Department of Engineering - Rome, Italy
}

\begin{abstract}
The optimal design methodologies in aeronautics are known to be constrained by the computational burden required by direct simulations. Due to this reason, the development of efficient metamodelling techniques represents nowadays an imperative need for the designers. In fact, surrogate models has been demonstrated to significantly reduce the number of high-fidelity evaluations, thus alleviating the computing effort. Over the last years, the aeronautical designers' community has switched from a design approach predominantly based on direct simulations to an extensive use of metamodels. Recently, to further improve the efficiency, several dynamic approaches based on self-tuning parameters have been developed to support the metamodel construction. This work deals with the use of surrogate models based on Artificial Neural Network for the noise shielding of unconventional aircraft configurations. Here, the insertion loss field of a 2D airfoil is reproduced through advanced machine learning techniques. The relevant framework is the calculation of the noise emitted by innovative aircraft configurations by means of suitable corrections of existing well-assessed noise prediction tools. The self-tuning algorithm has demonstrated to be accurate and efficient, and the observed performance discloses the possibility to implement numerical strategies for the reliable and robust unconventional aircraft optimal design.
\end{abstract}

\section{INTRODUCTION}

One of the main challenges for the aeronautical field nowadays is to support the constant increase in air traffic while assuring the environmental sustainability of the aviation, from the point of view of both noise signature and gas emissions. The current aircraft architectures have reached a state of technological saturation, implying the necessity for the introduction of breakthrough concepts to produce a significant improvement in their environmental sustainability. The unconventionality of new concepts makes the application of existing semi-empirical and analytical models almost impossible, thus requiring

\footnotetext{
*email:francesco.centracchio@uniroma3.it

†email: lorenzo.burghignoli@uniroma3.it

‡email:giorgio.palma@uniroma3.it

§email:ilaria.cioffi@uniroma3.it

I email: umberto.iemma@uniroma3.it
} 
computationally expensive direct numerical simulations since the conceptual design phase. This could lead to a prohibitive computational effort [1], not compatible with the timelines required to the designer. Thus, the Simulation Based Design Optimisation (SBDO), paradigm of complex industrial products, is moving towards a massive use of metamodels in many engineering branches. A sampling of the domain is performed to train an analytical model of the objective functions, and such a model is used by the optimiser to find the optimal solution of the problem. Metamodelling techniques demonstrated to be an efficient strategy in reducing the computational cost of SBDO, using only a small number of high-fidelity evaluations to create a surrogate model [2-5]. The resulting surrogate model preserves the information contained in the simulations, with the evaluation cost of an analytic function. To date, there exist a huge number of metamodelling techniques such as Kriging (KG) predictor, Radial Basis Functions (RBF), Support Vector Regression (SVR), Gaussian processes (GPR), etc. The recent availability of wide computing resources brought to a massive use of Artificial Neural Networks (ANN) in surrogate modelling. The idea of triggering a dichotomy between the biological neuron and mathematical processes has long historical roots [6,7], and the theoretical layout of complex multilayer structures has been investigated in the last 50 years $[8,9]$. Recently, the engineering community has developed several strategies aimed at improving the efficiency of the metamodelling process, by means of dynamic approaches based on selftuning techniques and adaptive sampling schemes $[4,10]$. The metamodelling strategy illustrated so far is applied to assess the acoustic signature of a Blended Wing Body (BWB), a tailless unconventional aircraft concept belonging to the flying-wing category [11], characterised by a large centre-body section shaped like an airfoil contributing to the overall lift generation, and accommodating the payload. This concept offers an interesting possibility from an acoustic point of view, allowing the installation of the engines above the lifting surface, thus providing a shielding of the noise directed towards the ground [12-14].

This work is organised as follows: Section 2 is dedicated to the description of the metric used to quantify the noise shielding effect; a brief outline on the dynamic ANN algorithm is reported in Sec. 3; Section 4 describes the benchmark case study analysed here, and in Sec. 5 are reported the numerical results; lastly, Sec. 6 gathers some concluding remarks.

\section{NOISE SHIELDING METRIC}

The possibility to mount the engines on top of the centre-body lifting surface is one of the main peculiarity of Blended-Wing-Body layouts. Due to the shielding effect, the sound energy of some engine noise components turns out to be less intense if compared to that of conventional aircraft configuration with engines mounted under the wing. The metric used for the noise shielding effect characterisation is the Overall Normalized Shielding Level $\bar{\gamma}[15]$. It is defined, in the frequency domain, as it follows

$$
\begin{aligned}
& \bar{\gamma}(\mathbf{x})=20 \log _{10} \bar{\eta}(\mathbf{x}) \\
& \bar{\eta}(\mathbf{x})=\sqrt{\frac{1}{\left(f_{u}-f_{l}\right)} \int_{f_{l}}^{f_{u}} \eta^{2}(\mathbf{x}, f) d f} \\
& \eta(\mathbf{x}, f)=\frac{\left|\tilde{p}_{T}(\mathbf{x}, f)\right|}{\left|\tilde{p}_{I}(\mathbf{x}, f)\right|}
\end{aligned}
$$

where $\tilde{p}_{I}$ is the incident pressure, $\tilde{p}_{T}=\tilde{p}_{I}+\tilde{p}_{S}$ the total pressure (with $\tilde{p}_{S}$ the scattered pressure), $f_{l}$ and $f_{u}$ are the lower and upper bounds of the considered frequency band. Pressure data are computed with direct simulations by means of the integral representation of the 2D Helmholtz equation.

\section{NEURAL NETWORKS DYNAMIC METAMODELLING}

The goal of a metamodel is to catch the fundamental dynamics of the data (provided by experiments or simulations), by means of the response of an analytical model. Let $\mathcal{T}$ be the training set composed by $M$ 
points $\left\{\mathbf{x}_{i}\right\}_{i=1}^{M}$, with associated $M$ functions vectors $\left\{\mathbf{y}_{i}\right\}_{i=1}^{M}$. The ANN response is able to quickly predict function values $\hat{y}_{i j}$ related to a generic $\mathbf{x}_{i}$ belonging to the domain $\mathcal{D}$.

Artificial neural networks are based on the concept of artificial neuron: it is a simple mathematical structure which accepts several inputs and a single output. The neuron output $a_{k}=f\left(z_{k}\right)$, with $f_{l}$ the activation function, is computed starting from the nodal activation potential $z_{k}=\sum_{j=1}^{n} w_{j k} x_{j}+b_{k}$ (with $w_{j k}$ the synaptic weight of the $j$-th input to the $k$-th node, $x_{j}$ the node input and $b_{k}$ the bias, an additional parameter required to adjust the output). Let now consider a multilayer structure composed by $L=Q+2$ layers, i.e. the input layer (with $N_{i}$ input neurons), $\mathbf{N}_{\mathbf{h}}=\left(N_{h_{1}}, \ldots, N_{h_{Q}}\right)$ hidden neurons organised in $Q$ hidden layers, and the output layer (composed by $N_{o}$ output neurons): the input-output functional relation for each layer can be written in compact form as it follows

$$
\mathbf{a}_{l}=\mathbf{f}_{l}\left(\mathbf{W}_{l-1} \mathbf{a}_{l-1}+\mathbf{b}_{l}\right)
$$

so that the inputs $\mathbf{x} \equiv \mathbf{a}_{1}$ and the outputs $\mathbf{y} \equiv \mathbf{a}_{L}$. The selection of weights matrices $\left\{\mathbf{W}_{l}\right\}_{l=1}^{L-1}$ and biases vectors $\left\{\mathbf{b}_{l}\right\}_{l=2}^{L}$ is performed here with the backpropagation technique. The input $\mathbf{x}$ is fed to the network and the the output is evaluated with the Eq. (2) together with an estimate of the error function $E_{t}$, defined as it follows

$$
E_{t}=\frac{1}{2} \sum_{i=1}^{R}\left(\mathbf{d}_{i}-\mathbf{y}_{i, t}\right)^{2}
$$

with $R$ the number of training set patterns, $\mathbf{d}_{i}$ the desired output vector and $\mathbf{y}_{i, t}$ the network output at the $t$-th epoch, both related to the $i$-th training pattern. Afterwards, the gradient of the loss function $\nabla E\left(\mathbf{d}_{i}, \mathbf{y}_{i, t}\right)$ is evaluated as a series of local intermediate gradients: the derivatives of $E$ with respect to weights and biases are evaluated backpropagating the error information starting from the output layer up to the input one. Eventually, the network parameters are modified based on the information retrieved: weights and biases at the $t$-th epoch are updated by means of the error gradient components scaled by the learning rate $\eta$, a free parameter selected prior to the training. As a result of the training process, the network is completely identified by its hyperparameters $\Theta=\left\{\mathbf{f}_{l}, \mathbf{W}_{l-1}, \mathbf{b}_{l}\right\}_{l=2}^{L}$, and the the network output is such that $\hat{\mathbf{y}}_{\mathbf{i}}$ is the image of $\mathbf{x}_{i}$. Within the training process, the average value of the Root Mean Squared Error (RMSE) evaluated at the $t$-th epoch is used as stop criterion

$$
e_{t}=\frac{1}{N_{y}} \sum_{j=1}^{N_{y}} \sqrt{\frac{\sum_{i=1}^{M}\left(y_{i j}-\hat{y}_{i j, t}\right)^{2}}{M}}
$$

being $N_{y}$ the size of the output vector, $M$ the size of $\mathcal{T}, \hat{y}_{i j}$ the prediction of the $j$-th output component over the $i$-th training point and $y_{i j}$ the true value.

\subsection{Hyperparameters selection}

The ANN fitting capability is sensitive to the network architecture: unfortunately, the set of topologies capable to correctly fit the training data is strongly problem dependent and there is not a specific scheme to find the optimal or near-optimal number of hidden layers and nodes. The active approach used here is capable to automatically select the architecture that ensures the desired fitting accuracy. The dynamic algorithm is based on the automatic selection of learning rate, activation functions and hidden topology, and the self-tuning scheme is driven by an early stopping criterion. The main phases of the algorithm are summarised below.

Learning rate update This feature aims at mitigating the effects of instabilities generated by large values of $\eta$. The training instabilities are detected by analysing in runtime the second derivative $\ddot{e}_{t}$ of the RMSE: when a change in the concavity of $e_{t}$ occurs, two consecutive values of $\ddot{e}_{t}$ turn out to have different signs, and it is convenient to decrease the learning rate with the aim of moving away from the instability. 
Early stopping The first derivative $\dot{e}_{t}$ of the Eq. (4) is a good candidate to foresee the fitting capabilities of the network: defining a patience parameter $k_{p}$, if $\dot{e}_{t}$ assumes near-zero values or strictly positive values, for a number of epochs equal to $k_{p}$, it means that the the prediction at the training points is not improving or is moving away from the true values respectively. In such events, the hidden topology is changed, and the training process starts again with a new architecture.

Activation functions selection The best combination of hidden and output layers activation function is automatically selected on the basis of a preliminary factorial tournament of the possible functions combinations. Within the tournament, for each couple of function to be analysed, the number of training epochs is provided by the patience parameter $k_{p}$. The initial tournament implies the additional computational cost $O\left(k_{p} N_{f}^{2}\right)$, with $N_{f}$ the number of candidate functions, which is recovered by a faster network training, due to the fact that the activation functions are the best performing for the specific problem.

Topology optimisation In the event that the training process exits due to the early stopping criterion, a new architecture is analysed and the training process starts again with the new topology. The in-house topology optimiser is fully deterministic, and each iteration of the algorithm mainly consists of the following steps:

- Selection of the hidden layers number and layers initialisation;

- Search close to the global minimum;

- Mutations of the worst architectures and new point addition.

It is worth mentioning that several deterministic initialisation schemes can be used for the first phase of the optimisation algorithm. Different schemes imply different convergence rates, and it has been noted that convergence speed seems to be conflicting with the network compactness. This behaviour is currently under investigation.

\subsection{Dynamic sampling}

The active training described so far leads to the definition of $\Theta$, that completely identify the dynamics of the available data: accordingly, given a generic point $\mathbf{x}$ in the design space, $\mathbf{y}=\mathcal{N}(\mathbf{x})$, with $\mathcal{N}$ the metamodel provided by the ANN. The adaptive sampling scheme used here aims at driving the designer towards the choice of new training points. The definition of a merit function representing the behaviour of the metamodel uncertainty plays a key role: the uncertainty must equals zero if evaluated at the training points, since the prediction accuracy set by the designer is ensured by current topology. Here, the uncertainty function is supposed to be linked to the spatial correlation between the elements of the set containing the training points $\left\{\mathbf{x}_{i}\right\}_{i=1}^{M}$ with associated the predictions $\left\{\hat{\mathbf{y}}_{i}\right\}_{i=1}^{M}$. To perform the adaptive sampling, the new training point is selected as it follows

$$
\mathbf{x}_{\text {new }}=\arg \max _{\mathbf{x} \in \mathcal{D}}[U(\mathbf{x})]
$$

so that the size of the training set is systematically increased, and a new network can be built using the active scheme described above.

\section{CASE STUDY}

The method presented in the previous sections is used in the following to build a surrogate model of noise shielding for the application case of a NACA0012 airfoil impinged by an incident field generated by a point source monopole placed on top of it, as depicted in Fig. 1. 


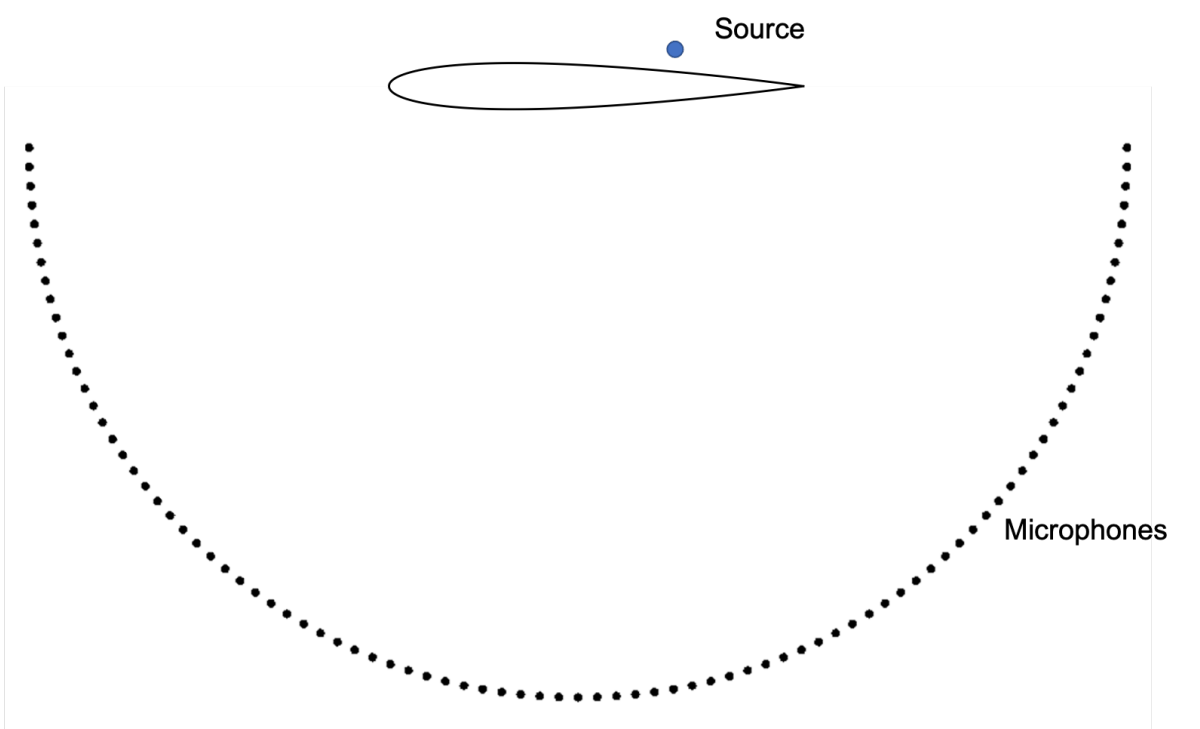

Figure 1: Sketch of the case study: the acoustic point source is placed over a unitary chord NACA 0012. The shielding metric $\bar{\gamma}$ is evaluated over an arc of virtual microphones.

Since this noise signal presents a rich spectral content, the quantities involved in the evaluation of the masking effect of the obstacle must take into account the contribution on the overall shielding effect of each spectral component and provide an estimate of the level reduction (or amplification) at specified locations induced by the scattering object in a clearly reproducible way. Such indicators have been defined in Sec. 2, being suitable to refer to the spectral characteristics of the masking effect of an obstacle on the field generated by a point source.

All the quantities are evaluated and assessed using a NACA 0012 airfoil, representing the center body section of the BWB, impinged by the acoustic field generated by an isotropic point source. Four positions of this source have been considered in the construction of the training set: the source is horizontally placed at $0.7,0.9,1.1$ and 1.3 times the chord from the leading edge of the airfoil, and vertically elevated the maximum thickness of the airfoil from the chord line. The value of the shielding metric $\bar{\gamma}(\mathbf{x})$ has been evaluated on a semicircle of 500 microphones located under the airfoil with a radius of 20 chords centred in the middle of the airfoil, integrated on the reduced frequency range $k c=1.83-36.63$, corresponding to $100-2000 \mathrm{~Hz}$ for unitary airfoil chord.

The metamodel produced in the present work is designed to take into account the shielding effect of the innovative architecture in the far-field by applying a correction directly to the source, specifically to its directivity and intensity. To correctly quantify the filter is important to consider a distance between the source and the microphones capable to guarantee the decay of the near-field terms. As evidenced in Sec. 2, the effects of the flow are not taken into account and the filters are assumed to be capable of correcting the noise field in static conditions.

\section{RESULTS}

In the application presented here, the true function, presented in Fig. 2, is assumed to be known and is used as validation set.

The accuracy on the training set is set at $0.5 \%$, whereas the stop criterion is based on the root-meansquare error RMSE between the $N_{v}$ elements of the validation set and the metamodel prediction. For each validation point, the normalised RMS error is computed as follows 


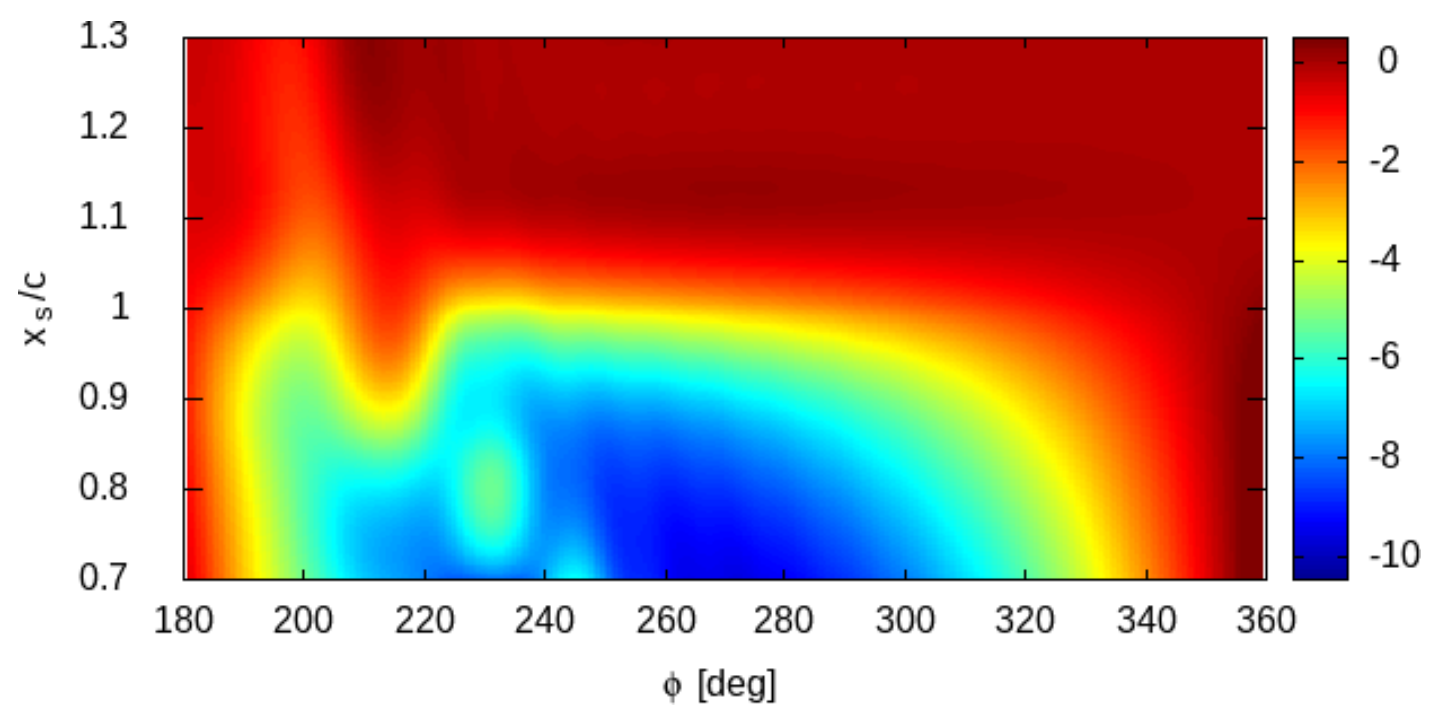

Figure 2: Overall normalised shielding level $\bar{\gamma}$ as a function of the polar directivity angle $\phi$ and nondimensional source location $x_{s} / c$ : true function.

$$
R M S E(\mathbf{x})=\sqrt{\frac{1}{N_{v}} \sum_{i=1}^{N_{v}}\left[\frac{f\left(\mathbf{x}_{i}\right)-\hat{f}\left(\mathbf{x}_{i}\right)}{\max (f)-\min (f)}\right]^{2}}
$$

and the target was set at $1 \%$. The trends of the RMSE and of the uncertainty function, both as a function of the points added to the TS, is shown below in Fig. 3.

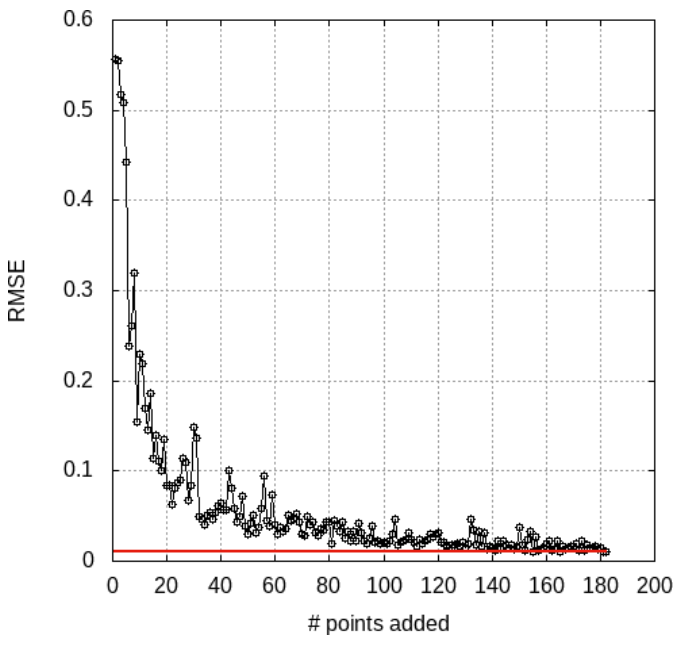

(a) RMS error

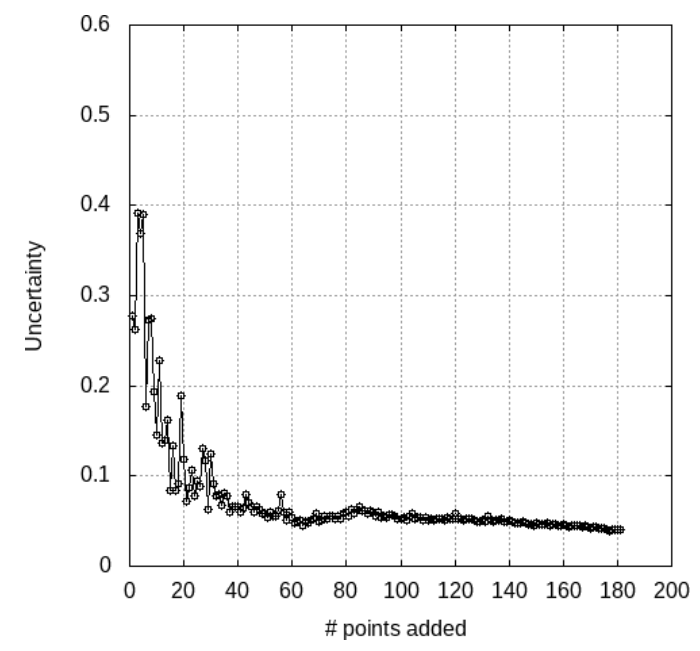

(b) Uncertainty

Figure 3: Convergence as a function of the new training points added.

As shown in Fig. 3a, the dynamic neural network successfully converges to the $E_{R M S}$ target value by adding 181 points to the initial TS: the convergence, despite locally irregular, turns out to have an overall decreasing behaviour. Figure $3 b$ shows that the uncertainty trend is also globally decreasing (except for the 
region around the 70-th point added). Nevertheless, this result is encouraging because it makes it possible to assess a stop criterion based on the uncertainty in real-life applications, i.e. when the true function is unknown. The optimal topology has three hidden layers (with 47, 19 and 14 neurons) with gaussian activation on the hidden layers and hyperbolic tangent on the output layer. The prediction of resulting metamodel is shown in Fig. 4 along with the final distribution of the TS points.

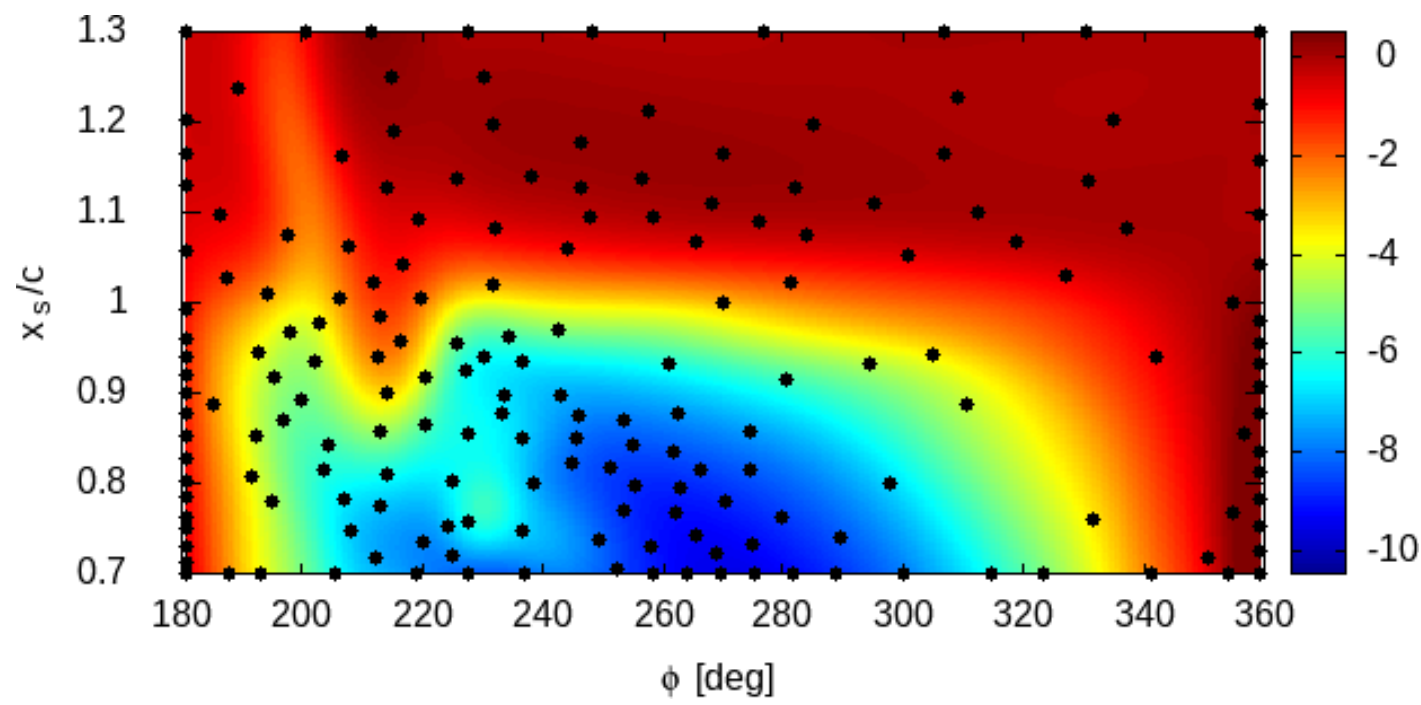

Figure 4: $\bar{\gamma}$ as a function of the polar directivity angle $\phi$ and non-dimensional source location $x_{s} / c$ : ANNbased metamodel response and final training set (black dots).

Figure 4 shows that the final metamodel prediction is in excellent agreement with the true function in Fig. 2 in both the function shape and the absolute values: this demonstrates the effectiveness of the developed technique. The map of the absolute error (see Fig. 5) is depicted below, in Fig. 5.

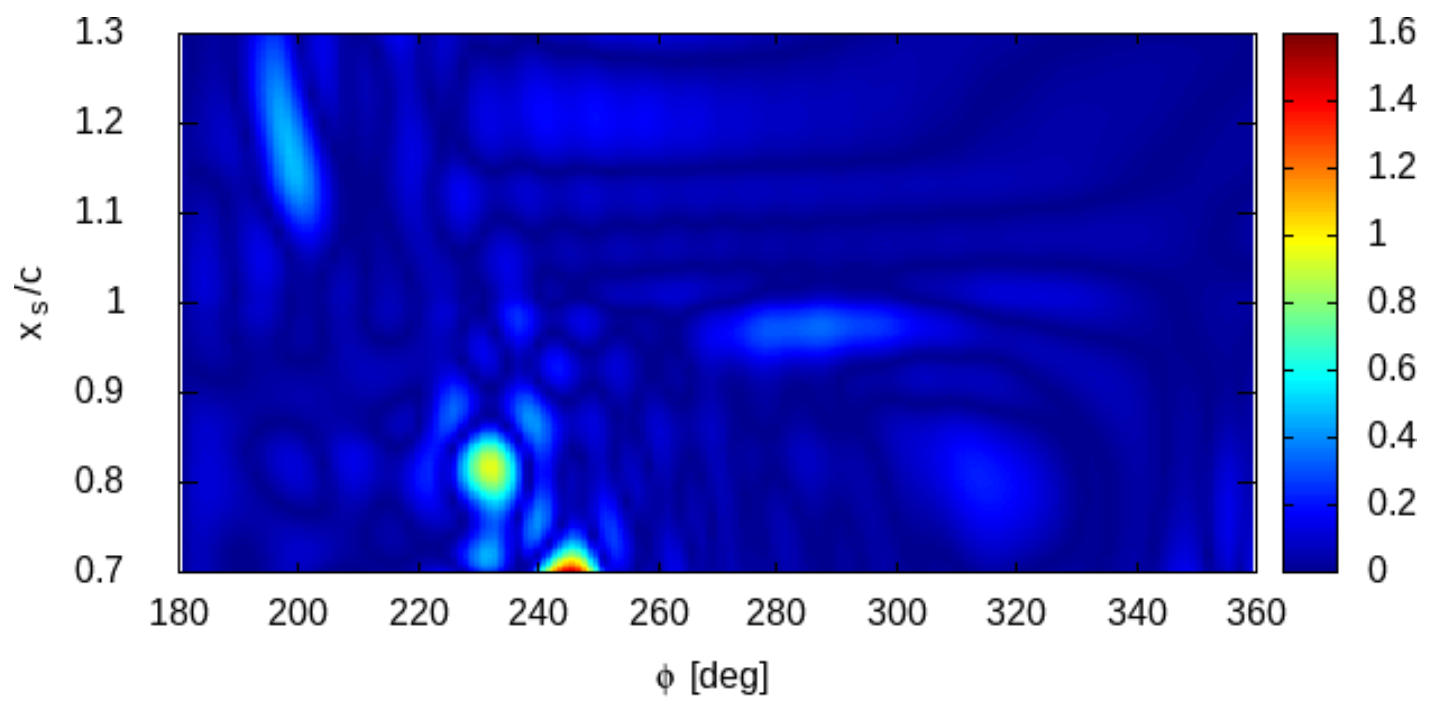

Figure 5: Absolute error of the ANN-based metamodel with respect to the true function.

Fig. 5 shows two regions in which the values are of the order of $1 \mathrm{~dB}$ and $1.6 \mathrm{~dB}$ respectively. In the 
remaining part of the domain the absolute error ranges between about 0.2 and $0.5 \mathrm{~dB}$, confirming the excellent accuracy of the metamodel. The highest values of the absolute error are found in correspondence with the lowest values of the uncertainty of the metamodel, presented in Fig. 6.

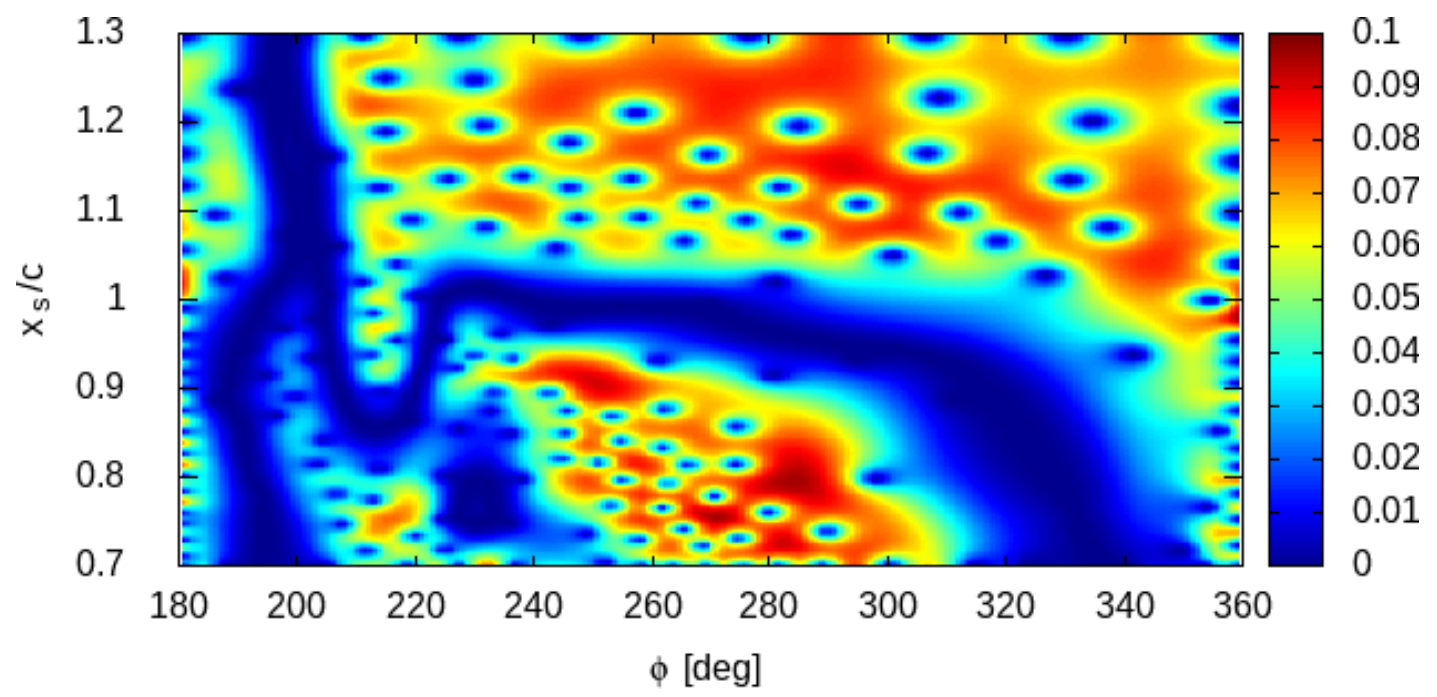

Figure 6: Uncertainty based on spatial correlation related to the ANN-based metamodel.

As expected, the uncertainty map of Fig. 6 shows zero uncertainty zones at the training points location, connected to each other by bumps that can exhibit one or more local maxima of uncertainty.

\section{CONCLUSIONS}

The paper presents an original metamodelling technique based on dynamic Artificial Neural Networks for aeronautical applications. The proposed method features self-tuning and adaptive sampling strategies to dynamically improve the metamodel accuracy. Starting from an initial set based on few simulations, the Training Set is enriched at each iteration with new points where the knowledge of the target response needs to be improved. Specifically, the uncertainty based on the spatial-correlations between the TS points is used to drive the adaptive process. The case study presented here is related to the modelling of the overall noise shielding factor for Blended-Wing-Body aircraft layouts. In particular the selected metric has been evaluated for a NACA 0012 airfoil, representing the center body section of the BWB, impinged by the acoustic field generated by an isotropic point source. In the construction of the training set, four source positions have been considered. The shielding factor has been evaluated on a semicircle of 500 microphones located under the airfoil placed at 20 chords centred in the middle of the airfoil: the local values of the shielding factor have been integrated on the reduced frequency range $k c=1.83-36.63$, corresponding to $100-2000 \mathrm{~Hz}$ for unitary airfoil chord. Results have shown that the final metamodel prediction is in excellent agreement with the true function in both the function shape and the absolute values. In addition, it is worth noting that the uncertainty trend is globally decreasing, thus allowing its use for the definition of a stop criterion in real-life applications.

\section{ACKNOWLEDGEMENTS}

This work has been supported by the European Union Horizon 2020 research and innovation programme under project ANIMA (Aviation Noise Impact Management through Novel Approaches), grant agreement No. 769627. 


\section{REFERENCES}

[1] Anthony A. Giunta and Layne T. Watson. Using surrogate models and response surfaces in structural optimization with application to crashworthiness design and sheet metal forming. Technical report, 1998.

[2] Lorenzo Burghignoli, Francesco Centracchio, Umberto Iemma, and Monica Rossetti. Multi-objective optimization of bwb aircraft for noise shielding improvement. In Proceedings of the 25th International Congress on Sound and Vibration (ICSV 25), Hiroshima, Japan, 2018.

[3] Francesco Centracchio, Lorenzo Burghignoli, Umberto Iemma, and Monica Rossetti. Noise shielding models for the conceptual design of unconventional aircraft. In Proceedings of the 47th International Congress and Exposition on Noise Control Engineering (inter-noise2018), Chicago, Illinois, 2018.

[4] Umberto Iemma, Lorenzo Burghignoli, and Monica Rossetti. Radial basis functions for stochastic metamodels tailored to aeroacoustic applications. In 25th AIAA/CEAS Aeroacoustics Conference, Delft, The Netherlands, 2019.

[5] Lorenzo Burghignoli, Monica Rossetti, Francesco Centracchio, and Umberto Iemma. Noise shielding metamodels based on stochastic radial basis functions. In Proceedings of 26th International Congress on Sound and Vibration (ICSV 26), Montreal, Canada, 2019.

[6] Warren McCulloch and Walter Pitts. A logical calculus of the ideas immanent in nervous activity. The bulletin of mathematical biophysics, 5:115-133, 1943.

[7] Donald Hebb. The Organization of Behaviour. John Wiley \& Sons, Inc., 1949.

[8] A.G. Ivakhnenko. Cybernetics and forecasting techniques. Elsevier Science Ltd, 1967.

[9] A.G. Ivakhnenko. Cybernetic predicting devices. CCM Information Corporation, 1973.

[10] Silvi Volpi, Matteo Diez, Nicholas J. Gaul, Hyeongjin Song, Umberto Iemma, K.K. Choi, and Frederick Campana, Emilio F.and Stern. evelopment and validation of a dynamic metamodel based on stochastic radial basis functions and uncertainty quantification. Structural and Multidisciplinary Optimization, 51:347-368, 2015.

[11] Francesco Centracchio, Monica Rossetti, and Umberto Iemma. Approach to the weight estimation in the conceptual design of hybrid-electric-powered unconventional regional aircraft. Journal of Advanced Transportation, page 15, 2018.

[12] Giorgio Palma, Francesco Centracchio, and Lorenzo Burghignoli. Optimized metamaterials for enhanced noise shielding of innovative aircraft configurations. In Proceedings of the 27th International Congress on Sound and Vibration (ICSV 27), Virtual congress, 2021.

[13] Lorenzo Clark and Carl Gerhold. Inlet noise reduction by shielding for the blended-wing-body airplane. In 5th AIAA/CEAS Aeroacoustics Conference and Exhibit, 1999.

[14] Yohei Katsurayama and Taro Imamura. Numerical simulation of engine noise shielding around blended wing body aircraft. Transactions of the Japan society for aeronautical and space sciences, 58(2):83-88, 2015.

[15] Karl-Stéphane Rossignol, Jan Delfs, Michael Mößner, Denis Gély, Jean Bulté, and Florence V Hutchson. Experimental investigations on noise shielding: dependency on reference noise source and testing environment. In 2018 AIAA/CEAS Aeroacoustics Conference, page 2820, 2018. 